\title{
Long non-coding RNA SNHG12 promotes proliferation and invasion of colorectal cancer cells by acting as a molecular sponge of microRNA-16
}

\author{
YUEHUA LIU, JINGYU ZHOU, SHALONG WANG, YULIANG SONG, JIANPING ZHOU and FENG REN \\ Department of Geriatric Surgery, The Second Xiangya Hospital, Central South University, \\ Changsha, Hunan 410011, P.R. China
}

Received August 19, 2018; Accepted April 18, 2019

DOI: $10.3892 /$ etm.2019.7650

\begin{abstract}
Long non-coding (lnc)RNA small nucleolar RNA host gene 12 (SNHG12) has an oncogenic role in various common human cancer types, including colorectal cancer (CRC). However, the detailed regulatory mechanisms of SNHG12 in CRC cells have remained largely elusive, and the investigation thereof was the purpose of the present study. Polymerase chain reaction analysis was performed to examine the expression of IncRNA and microRNA (miR). Cell Counting Kit-8 and Transwell assays were used to assess cell proliferation and invasion. A luciferase reporter assay was performed to confirm a predicted targeting association between lncRNA and miR. It was observed that SNHG12 was markedly upregulated in CRC tissues when compared with that in adjacent non-tumour tissues, and its high expression was associated with CRC progression, as well as poor prognosis of patients. In addition, the expression of SNHG12 was higher in CRC cell lines when compared with that in a normal intestinal epithelial cell line. Knockdown of SNHG12 significantly inhibited CRC cell proliferation and invasion, while ectopic overexpression of SNHG12 had the opposite effect. A Bioinformatics analysis predicted that SNHG12 and miR-16 have complementary binding sites, which was confirmed by a luciferase reporter gene assay. The expression levels of miR-16 were markedly decreased in CRC tissues and cell lines compared with those in normal tissues or cells, and were inversely correlated with the expression levels of SNHG12 in CRC tissues. Furthermore, silencing of miR-16 eliminated the suppressive effects of SNHG12 knockdown on CRC cell proliferation and invasion. In conclusion, the present study demonstrated that SNHG12
\end{abstract}

Correspondence to: Professor Feng Ren, Department of Geriatric Surgery, The Second Xiangya Hospital, Central South University, 139 Middle Renmin Road, Changsha, Hunan 410011, P.R. China E-mail: renfeng@csu.edu.cn

Key words: long non-coding RNA, microRNA, proliferation, invasion, colorectal cancer, small nucleolar RNA host gene 12, microRNA16 promotes CRC cell proliferation and invasion, at least in part, by acting as a molecular sponge of miR-16, suggesting that SNHG12 may be a promising therapeutic target for CRC.

\section{Introduction}

Colorectal cancer (CRC) is one of the most common types of human malignancy worldwide (1). In recent decades, the combined treatment for CRC has improved, which includes surgical resection, radiotherapy and chemoradiotherapy; however, the prognosis of patients with advanced CRC remains poor (2). It has been demonstrated that CRC is driven by a series of successive genetic and epigenetic changes, which cause malignant transformation and metastasis (3-5). Non-coding RNAs are important epigenetic factors associated with cancer development (6-10), but the functions of various non-coding RNAs in CRC remain to be fully elucidated.

Long non-coding RNAs (lncRNAs), a class of non-coding RNAs longer than 200 nucleotides, are 10-fold lower in abundance than mRNAs (11). It has been demonstrated that there are thousands of IncRNA small nucleolar RNA host gene 12 (SNHG12) sequences in mammals and that the majority of lncRNAs are likely to be functional; however, only a small proportion of lncRNAs have been studied (12). Through regulation of gene expression at transcriptional, post-transcriptional and epigenetic levels, lncRNAs have important roles in various physiological and pathological processes, including cell growth, proliferation, differentiation, apoptosis and tumourigenesis $(13,14)$. Numerous 1 chRAs have been reported to function as promoters during cancer development and progression $(15,16)$. Therefore, these lncRNAs may become promising biomarkers and/or therapeutic targets for various cancer types.

Recently, several studies have indicated that the lncRNA SNHG12 functions as an oncogene in certain, common cancer types, including osteosarcoma, hepatocellular carcinoma, breast cancer, lung cancer, gastric cancer, glioma, cervical cancer, papillary thyroid carcinoma and CRC (17-25). For instance, Lei et al (20) reported that SNHG12 promoted the proliferation and migration of glioma cells by binding to ELAV like RNA binding protein 1. Ding et al (21) indicated that SNHG12 enhanced the proliferation and metastasis of papillary thyroid carcinoma cells by regulating the $\mathrm{Wnt} / \beta$-catenin 
signalling pathway. Furthermore, Wang et al (17) reported that SNHG12 promotes the proliferation and inhibits apoptosis of CRC cells. However, the regulatory mechanisms of how SNHG12 affects CRC growth and metastasis have remained elusive.

MicroRNAs (miRs), a class of small non-coding RNAs of 22-25 nt in length, are key regulators of gene expression (26). They directly bind to the 3'-untranslated region (3'-UTR) of their target mRNAs to thereby cause translational repression or mRNA degradation (26). A large number of studies have reported that miRs have key roles in the development and progression of human cancer types, including CRC $(27,28)$. In recent years, Qian et al (29) reported that miR-16 was significantly downregulated in CRC tissues compared with that in normal colonic mucosa, and miR-16 expression was associated with tumour differentiation, lymph node metastasis, TNM stage and tumour recurrence in CRC. Furthermore, low miR-16 expression is an independent factor predicting a poor prognosis for CRC patients (29). In addition, miR-16 has been indicated to inhibit CRC cell growth in vitro by regulating the p53-survivin signalling pathway (30). However, the association between SNHG12 and miR-16 in CRC has remained elusive.

Therefore, the present study was performed to explore the association of SNHG12 and miR-16 in CRC and the underlying molecular mechanisms in CRC cells in vitro.

\section{Materials and methods}

Clinical tissue samples. A total of $53 \mathrm{CRC}$ tissues and their matched adjacent non-tumour tissues were obtained from primary CRC patients at the Second Xiangya Hospital, Central South University (Changsha, China) between April 2011 and June 2012. These 53 CRC patients included 31 males and 22 females aged between 34 to 71 years (mean age, 53.7 years). The tissue samples were immediately snap-frozen in liquid nitrogen after surgical resection and stored until use.

Cell culture. Normal intestinal epithelial NCM460 cells and CRC cell lines, including HT-29, SW480, SW620 and LOVO, were obtained from the Cell Bank of Central South University and were cultured in Dulbecco's modified Eagle's medium (Thermo Fisher Scientific, Inc.) with $10 \%$ foetal bovine serum (FBS; HyClone; GE Healthcare) in a humidified atmosphere with $5 \% \mathrm{CO}_{2}$ at $37^{\circ} \mathrm{C}$.

Cell transfection. For cell transfection, SW620 and HT-29 cells $\left(1 \times 10^{6}\right.$ per well) were seeded into 6 -well plates, cultured overnight and transfected with $100 \mathrm{nM}$ non-specific short interfering (si)RNA (NC siRNA; cat. no. 12935300; Thermo Fisher Scientific, Inc.), 100 nM SNHG12-specific siRNA (SNHG12 siRNA; cat. no. AM16708; Thermo Fisher Scientific, Inc.), 100 nM pcDNA3.1 vector (cat. no. V00008; Yearthbio,Inc.), $100 \mathrm{nM}$ pcDNA-SNHG12 expression plasmid (cat. no. P00951; Yearthbio, Inc.), 100 nM miR-16 inhibitor (cat. no. 4464084; Thermo Fisher Scientific, Inc.) and/or 100 nM negative control (NC) inhibitor (cat. no. 4464076; Thermo Fisher Scientific, Inc.) by using Lipofectamine ${ }^{\circledR}$ 2000 (Thermo Fisher Scientific, Inc.) according to the manufacturer's protocol. The subsequent experiments were performed a $48 \mathrm{~h}$ after transfection.
Reverse transcription-quantitative (RT-q)PCR. Total RNA was extracted from tissues and cells using TRIzol reagent (Thermo Fisher Scientific, Inc). To detect the expression of miR-16 or SNHG12, RNA was reverse-transcribed into complementary (c)DNA using the PrimeScript ${ }^{\mathrm{TM}}$ II 1st Strand cDNA Synthesis kit (Takara Bio, Inc.) according to the manufacturer's protocol. Subsequently, qPCR was performed using the SYBR Premix kit (Takara Bio, Inc.) or the SYBR Prime Script miRNA RT-PCR kit (Takara Bio, Inc.). U6 and GAPDH were used as internal references. The relative expression was determined using the $2^{-\Delta \Delta \mathrm{Cq}}$ method (31). The following primers were used: GAPDH forward, 5'-CTGGGCTAC ACTGAGCACC-3' and reverse, 5'-AAGTGGTCGTTGAGG GCAATG-3'; and SNHG16 forward, 5'-GTGCCTCAGGAA GTCTCTTGCC-3' and reverse, 5'-ATCCAAACAAGTTAT CACACAGCAC-3'. Primers for U6 (cat. no. HmiRQP9001) and miR-16 (cat. no. HmiRQP0226) were obtained from Guangzhou FulenGen, Co., Ltd.

Bioinformatics analysis and luciferase reporter gene assay. TargetScan (www.targetscan.org) software predicted that SNHG12 was a potential target gene of miR-16. SW620 and HT-29 cells were transfected with a wild-type (WT) or a mutant (MT) SNHG12 pMIR-REPORT ${ }^{\mathrm{TM}}$ miRNA Expression Reporter Vector (Ambion; Thermo Fisher Scientific, Inc.) containing a 4-bp mutation at the predicted miR-16 binding site within SNHG12, along with miR-16 mimics (cat. no. 4464066; Thermo Fisher Scientific, Inc.) or miR-NC mimics (cat. no. 4464058; Thermo Fisher Scientific, Inc.) using Lipofectamine 2000. After transfection for $48 \mathrm{~h}$, a luciferase reporter gene assay was performed using the Dual-Luciferase ${ }^{\circledR}$ Reporter Assay System (Promega Corporation). The activity of firefly luciferase was normalized to the activity of Renilla luciferase.

Cell Counting Kit (CCK)-8 assay. The transfected cells $(2,000$ cells/well) were seeded in 96 -well plates and cultured at $37^{\circ} \mathrm{C}$ with $5 \% \mathrm{CO}_{2}$ for $0,24,48,72$ and $96 \mathrm{~h}$, respectively. The cell proliferation was then assessed using a CCK-8 (Thermo Fisher Scientific, Inc.), according to the manufacturer's protocol. The optical density value was read at a wavelength of $450 \mathrm{~nm}$ using a microplate reader (Bio-Rad Laboratories, Inc.).

Cell invasion assay. Cell invasion was assessed using Transwell chambers pre-coated with Matrige ${ }^{\circledR}$ (BD Biosciences). The transfected cells $\left(1 \times 10^{5}\right.$ per chamber) were re-suspended in $300 \mu 1$ serum-free DMEM and added to the upper chamber, and the lower chamber was filled with medium containing $10 \%$ FBS. After incubation at $37^{\circ} \mathrm{C}$ for $48 \mathrm{~h}$, cells that had transgressed through the membrane were fixed with $37 \%$ methanol at room temperature for $30 \mathrm{~min}$, and stained with $0.5 \%$ crystal violet at room temperature for $10 \mathrm{~min}$. The invaded cells were counted under a microscope.

Statistical analysis. Values are expressed as the mean \pm standard deviation. SPSS 19.0 (IBM Corp.) was used for statistical analysis. An unpaired or paired Student's t-test was used for comparison between two groups. One-way analysis of variance followed by Tukey's post-hoc test was used for comparisons of more than two groups. A 
A

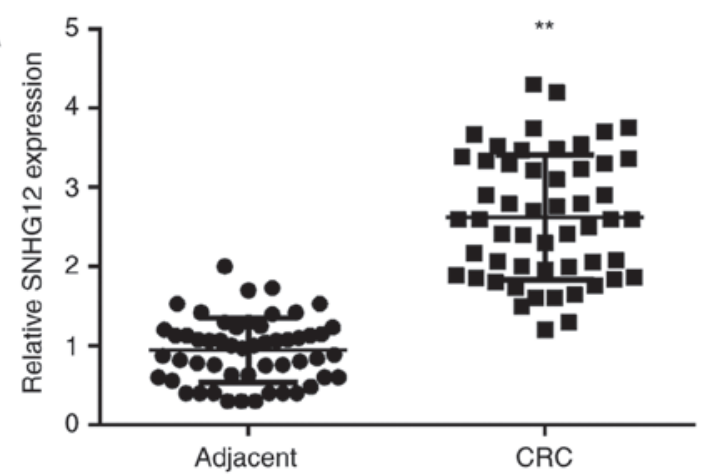

C

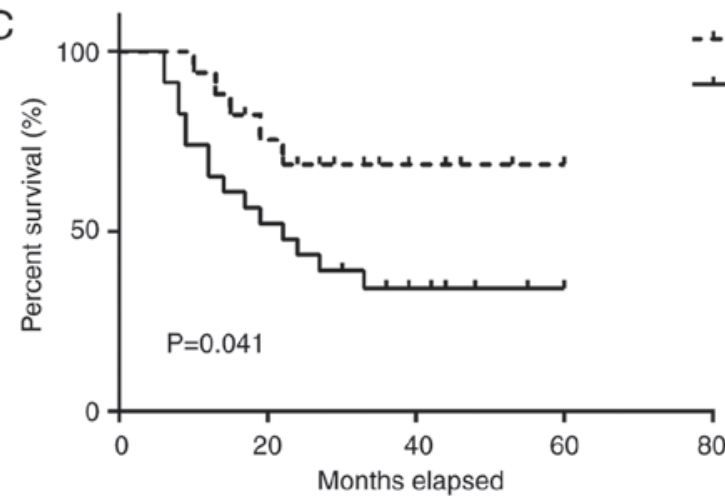

B

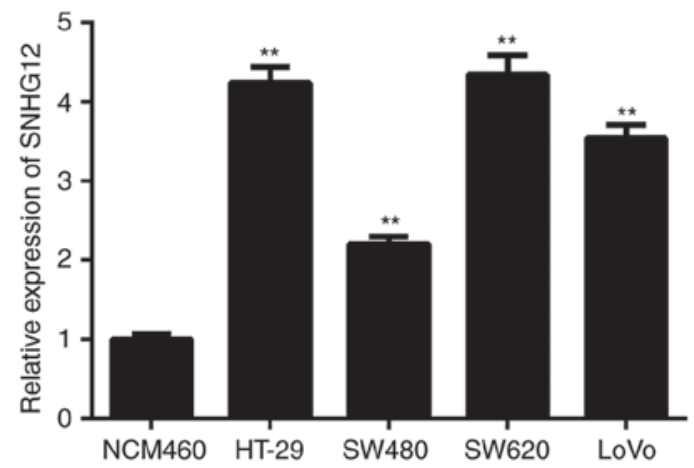

- ᄂ. Low SNHG12 expression

ـ High SNHG12 expression

Figure 1. SNHG12 is upregulated in CRC. (A) SNHG12 is upregulated in CRC tissues compared with that in adjacent non-tumour tissues. ${ }^{* *} \mathrm{P}<0.01$ vs. Adjacent. (B) SNHG12 is upregulated in CRC cell lines compared with that in NCM460 cells. ${ }^{* *} \mathrm{P}<0.01$ vs. NCM460 cells. (C) Kaplan-Meier analysis indicated that CRC patients with high SNHG12 expression had a significantly poorer overall survival when compared with that of the group with low SNHG12 expression. SNHG12, small nucleolar RNA host gene 12; CRC, colorectal cancer.

chi-square test was used to analyse the association between SNHG12 expression and clinicopathological characteristics in patients with CRC. Pearson correlation analysis was used to determine the association between miR-16 and SNHG12 expression in CRC tissues. The Kaplan-Meier method and a log-rank test were used to analyse the overall survival of patients with CRC. $\mathrm{P}<0.05$ was considered to indicate statistical significance.

\section{Results}

Upregulation of SNHG12 in CRC tissues and cell lines. First, the expression of IncRNA SNHG12 in CRC and adjacent non-tumour tissue samples was examined using RT-qPCR. As indicated in Fig. 1, the levels of SNHG12 were significantly higher in CRC tissues compared with those in adjacent non-tumour tissue samples. Consistently, the expression levels of SNHG12 were also markedly increased in CRC cell lines, including HT-29, SW480, SW620 and LOVO, when compared with those in NCM460 cells (Fig. 1B).

To further assess the clinical significance of SNHG12 expression in $\mathrm{CRC}$, the $\mathrm{CRC}$ patients were divided into high and low SNHG12 expression groups using the mean expression level of SNHG12 as the cut-off value. As presented in Table I, high expression of SNHG12 was significantly associated with lymph node metastasis and advanced clinical stage. Furthermore, the CRC patients with high SNHG12 expression had a poorer overall survival when compared with that in the group with low SNHG12 expression (Fig. 1C), suggesting that high SNHG12 expression may predict poor prognosis for CRC patients.
Function of SNHG12 in CRC cell proliferation and invasion. Next, the function of SNHG12 in the proliferation and invasion of CRC cells was examined. As the above results had indicated that SNHG12 was upregulated in CRC, SW620 and HT-29 cells were transfected with SNHG12 siRNA to reduce its expression. After transfection, the expression of SNHG12 was markedly downregulated in the siSNHG12 group when compared with that in the siNC group (Fig. 2A). A CKK-8 assay indicated that the proliferation of SW620 and HT-29 cells was significantly decreased in the siSNHG12 group compared with that in the siNC group, indicating that knockdown of SNHG12 expression inhibits CRC cell proliferation (Fig. 2B and C). Similarly, the Transwell assay suggested that the invasive capacity of SW620 and HT-29 cells was markedly decreased after silencing of SNHG12 expression (Fig. 2D).

To further confirm the above results, the effects of SNHG12 overexpression on the proliferation and invasion of CRC cells were then assessed. SW620 and HT-29 cells were transfected with SNHG12 plasmid or blank vector. After transfection, SNHG12 was significantly upregulated in the SNHG12 group when compared with that in the blank vector group (Fig. 2E). Furthermore, as indicated in Fig. 2F-H, the proliferation and invasion of SW620 and HT-29 cells was significantly increased in the SNHG12 group compared with that in the blank vector group. These results suggest that SNHG12 may have a role in promoting $\mathrm{CRC}$ growth and metastasis.

Targeting association between SNHG12 and miR-16 in CRC cells. Next, a Bioinformatics analysis was performed to identify miRs targeted by SNHG12, and it was indicated 
Table I. Association between SNHG12 expression and clinicopathological characteristics in patients with colorectal cancer $(n=53)$.

\begin{tabular}{|c|c|c|c|c|c|}
\hline \multirow[b]{2}{*}{ Variable } & \multirow[b]{2}{*}{$\mathrm{N}$} & \multicolumn{2}{|c|}{ SNHG12 expression } & \multirow[b]{2}{*}{ Chi-square value } & \multirow[b]{2}{*}{ P-value } \\
\hline & & Low $(n=27)$ & High $(n=26)$ & & \\
\hline Age (years) & & & & 0.930 & 0.335 \\
\hline$\leq 55$ & 27 & 12 & 15 & & \\
\hline$>55$ & 26 & 15 & 11 & & \\
\hline Sex & & & & 0.195 & 0.442 \\
\hline Male & 31 & 15 & 16 & & \\
\hline Female & 22 & 12 & 10 & & \\
\hline Differentiation & & & & 2.163 & 0.141 \\
\hline Well and moderate & 43 & 24 & 19 & & \\
\hline Poor & 10 & 3 & 7 & & \\
\hline Lymph node metastasis & & & & 2.419 & 0.016 \\
\hline Present & 18 & 5 & 13 & & \\
\hline Absent & 35 & 22 & 13 & & \\
\hline Tumor, nodes and metastasis stage & & & & 2.419 & 0.016 \\
\hline I-II & 35 & 22 & 13 & & \\
\hline III-IV & 18 & 5 & 13 & & \\
\hline
\end{tabular}

SNHG12, small nucleolar RNA host gene 12 .

that miR-16 and SNHG12 had complementary binding sites (Fig. 3A). Therefore, the expression levels of miR-16 in CRC tissue and cell lines were examined. As indicated in Fig. 3B and $\mathrm{C}$, the expression levels of miR-16 were markedly reduced in CRC tissues and cell lines when compared with those in adjacent non-tumour tissues and NCM460 cells, respectively. Furthermore, a significant inverse correlation was observed between SNHG12 and miR-16 expression in CRC tissues (Fig. 3D). To further confirm the association between SNHG12 and miR-16 in CRC, luciferase reporter plasmids containing WT or MT miR-16 binding sites of SNHG12 were constructed (Fig. 3E). The luciferase reporter assay indicated that transfection with miR-16 mimics markedly decreased the luciferase activity of the vector containing WT SNHG12 but had no effect on that in the vector carrying MT SNHG12 in SW620 and HT-29 cells (Fig. 3F-G), suggesting that SNHG12 acts as a sponge for miR-16 in CRC cells.

SNHG12 inhibits miR-16 expression in CRC cells. The effects of SNHG12 on miR-16 expression in CRC cells were then further studied. As presented in Fig. 4A, silencing of SNHG12 increased the expression of miR-16 in SW620 and HT-29 cells. Furthermore, overexpression of SNHG12 markedly reduced the expression of miR-16 in CRC cells (Fig. 4B). It was therefore indicated that SNHG12 negatively affects the miR-16 levels in CRC cells.

miR-16 is involved in SNHG12-induced CRC cell proliferation and invasion. Based on the above results, it was speculated that SNHG12 promotes CRC cell proliferation and invasion by acting as a sponge of miR-16. To validate this speculation, SW620 and HT-29 cells were co-transfected with NC
siRNA and NC inhibitor (siNC+NC in group), NC siRNA and miR-16 inhibitor (siNC+miR-16 in group), SNHG12 siRNA and NC inhibitor (siSNHG12+NC in group), or with SNHG12 siRNA and miR-16 inhibitor (siSNHG12+miR-16 in group). As presented in Fig. 5A, the expression levels of miR-16 were markedly lower in the siNC+miR-16 in group, while they were higher in the siSNHG12+NC group, when compared with those in the siNC+NC in group. Furthermore, the expression levels of miR-16 were lower in the siSNHG12+miR-16 in group when compared with those in the siSNHG12+NC in group. CKK-8 assay and Transwell assays further indicated that the proliferation and invasion of CRC cells were significantly reduced in the siSNHG12+NC in group when compared with those in the siNC+NC in group, while these effects were rescued in the siSNHG12+miR-16 in group (Fig. 5B-E). These results suggested that knockdown of SNHG12 inhibited CRC cell proliferation and invasion by upregulating miR-16.

\section{Discussion}

The regulatory mechanism of SNHG12 in CRC cells has remained largely elusive. In the present study, SNHG12 was identified to be markedly upregulated in CRC tissue when compared with adjacent non-tumour tissues, and its upregulation was markedly associated with CRC progression, as well as poor prognosis of patients. In addition, SNHG12 expression was higher in CRC cell lines when compared with that in normal intestinal epithelial cells. Knockdown of SNHG12 significantly repressed CRC cell proliferation and invasion, while SNHG12 overexpression significantly increased the proliferation and invasion of CRC cells. A Bioinformatics analysis indicated that SNHG12 and miR-16 

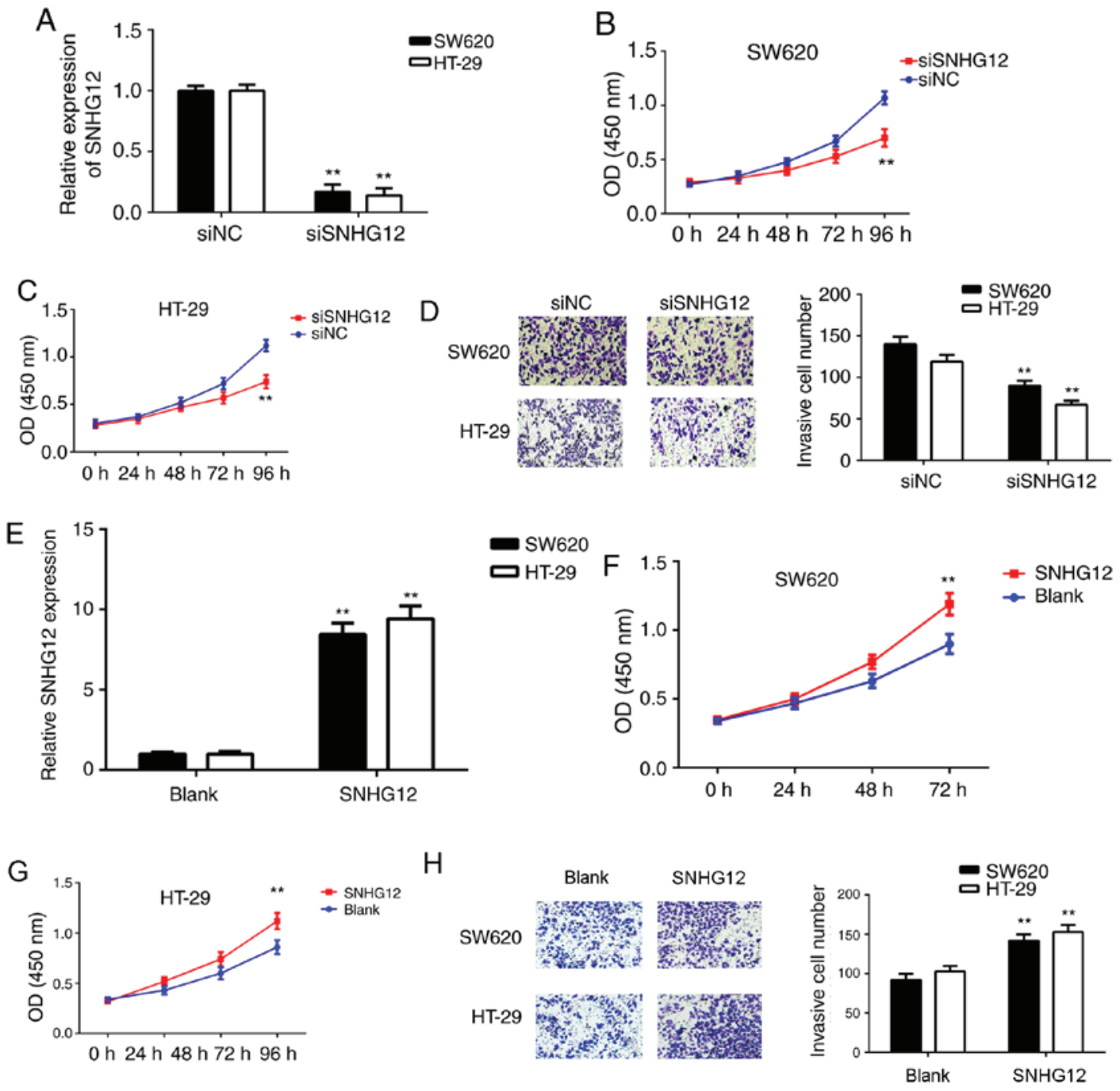

Figure 2. Inhibition of SNHG12 inhibits colorectal cancer cell proliferation and invasion. (A-D) SW620 and HT-29 cells were transfected with SNHG12 siRNA or NC siRNA, respectively. (A) RT-qPCR was used to examine the levels of SNHG12. CCK-8 assay using (B) SW620 and (C) HT-29 cells, and (D) a Transwell assay (magnification, $\mathrm{x} 200$ ) were performed to assess cell proliferation and invasion, respectively. For A-D, ${ }^{* *} \mathrm{P}<0.01 \mathrm{vs}$. NC siRNA. (E-H) Next, SW620 and HT-29 cells were transfected with SNHG12 expression plasmid or a blank vector, respectively. (E) RT-qPCR was used to determine the levels of SNHG12. CCK-8 assay using (F) SW620 and (G) HT-29 cells, and (H) a Transwell assay (magnification, x200) were performed to study cell proliferation and invasion. For E-H, ${ }^{* *} \mathrm{P}<0.01$ vs. Blank. Groups: siNC, cells transfected with NC siRNA; siSNHG12, cells transfected with siRNA targeting SNHG12; SNHG12, cells transfected with SNHG12 overexpression vector; Blank, cells transfected with empty vector. SNHG12, small nucleolar RNA host gene 12; CKK-8, Cell Counting Kit 8; siRNA, small interfering RNA; NC, negative control; OD, optical density; RT-qPCR, reverse transcription-quantitative polymerase chain reaction.

had complementary binding sites, which was confirmed by a luciferase reporter gene assay. The expression levels of miR-16 were markedly downregulated in CRC tissues and cell lines, and were inversely correlated with the expression of SNHG12 in CRC tissues. In addition, silencing of miR-16 eliminated the suppressive effects of SNHG12 downregulation on CRC cell proliferation and invasion.

It has been reported that SNHG12 has a role in promoting certain common human cancer types. For instance, SNHG12 has been indicated to promote cell proliferation and migration by increasing the expression of angiomotin in osteosarcoma cells (19). It was also reported to promote tumourigenesis and metastasis by targeting miR-199a/b-5p in hepatocellular carcinoma (18). Furthermore, SNHG12 contributes to multidrug resistance by activating the MAPK/Slug pathway by sponging miR-181a in non-small-cell lung cancer (32). In addition, C-MYC-induced upregulation of lncRNA SNHG12 promoted cell proliferation and migration, while inhibiting cell apoptosis in triple-negative breast cancer (25). Thus, SNHG12 has been suggested as a promising therapeutic target for specific cancer types $(18,32,33)$. Furthermore, knockdown of SNHG12 was demonstrated to inhibit non-small cell lung cancer cell growth and induce apoptosis by upregulating miR-138 (33). Recently, Wang et al (17) reported that SNHG12 promotes the proliferation and inhibits apoptosis of CRC cells. However, the underlying mechanisms of the regulatory function of SNHG12 during CRC growth and metastasis has remained elusive. The present study confirmed that the expression of SNHG12 was markedly upregulated in CRC tissues and cell lines when compared with that in adjacent normal tissues and normal intestinal epithelial cells, respectively. Consistent with the present results, Wang et al (17) also observed an increased expression of SNHG12 in CRC tissues and cell lines. Furthermore, the present study revealed that high expression of SNHG12 was 
A Wild type SNHG12 5'...TAGTCGCTGCTGCTG...3'<smiles>C1#CC#C1</smiles>

miR-16 $3^{\prime}$...TAAATGCACGACGAT... $5^{\prime}$

Mutant type SNHG12 $5^{\prime}$...TAGTCGCACGACGAG... $3^{\prime}$
$\mathrm{B}$

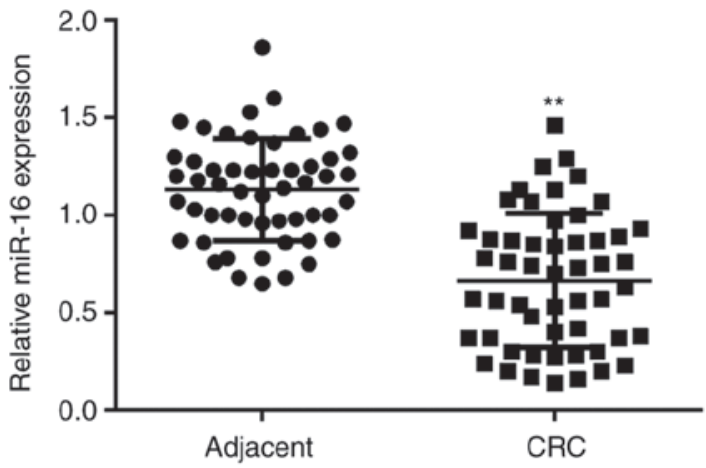

C

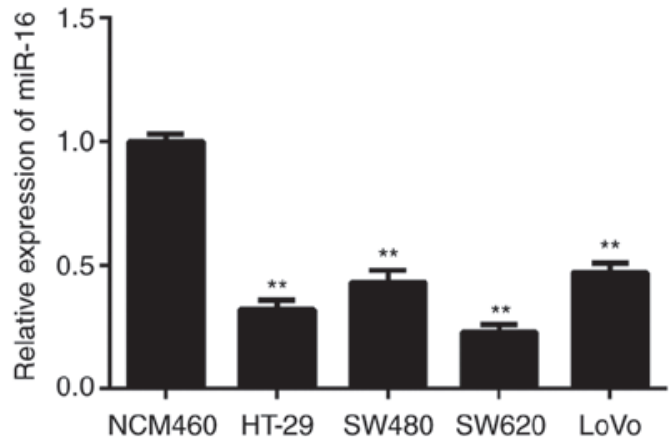

E

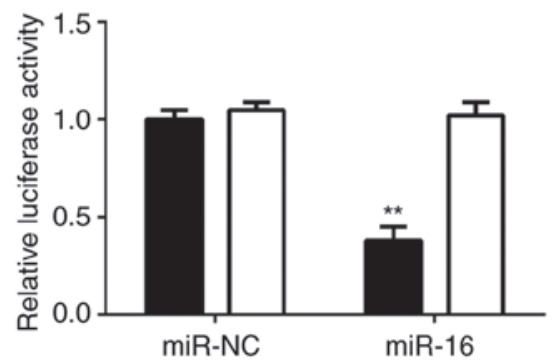

WT SNHG12 口MT SNHG12

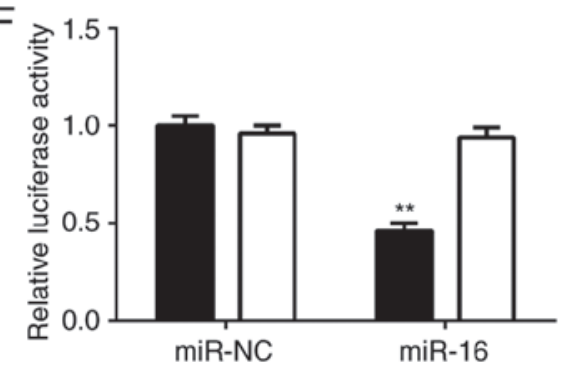

Figure 3. Targeting association between SNHG12 and miR-16 in CRC cells. (A) miR-16 was predicted to bind to SNHG12 by a Bioinformatics analysis (B) miR-16 is downregulated in CRC tissues compared with that in adjacent non-tumour tissues. ${ }^{* *} \mathrm{P}<0.01$ vs. Adjacent. (C) miR-16 is downregulated in CRC cell lines compared with that in NCM460 cells. " $\mathrm{P}<0.01$ vs. NCM460 cells. (D) Inverse correlation between SNHG12 and miR-16 expression in CRC tissue. (E) A luciferase reporter plasmid containing WT or MT miR-16 binding sites of SNHG12 was constructed. A luciferase reporter gene assay was performed in (E) SW620 and (F) HT-29 cells by co-transfection of the respective reporter plasmids and miR-16 mimics or miR-NC. WT, wild-type; MT, mutant type; miR, microRNA; SNHG12, small nucleolar RNA host gene 12; NC, negative control; CRC, colorectal cancer.
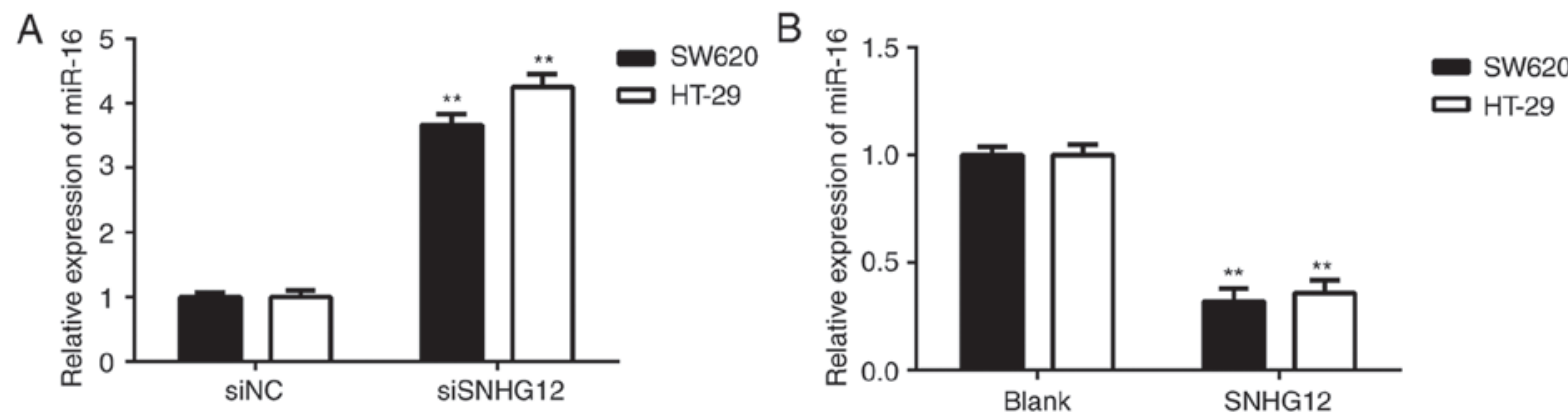

Figure 4. SNHG12 inhibits miR-16 expression in CRC cells. (A) Silencing of SNHG12 increased the expression of miR-16 in SW620 and HT-29 cells. ${ }^{* *} \mathrm{P}<0.01$ vs. NC siRNA. (B) SW620 and HT-29 cells were transfected with SNHG12 expression plasmid or a blank vector, respectively. The expression of miR-16 was then examined using reverse transcription-quantitative polymerase chain reaction. ${ }^{* *} \mathrm{P}<0.01 \mathrm{vs.} \mathrm{Blank.} \mathrm{Groups:} \mathrm{siNC,} \mathrm{cells} \mathrm{transfected} \mathrm{with} \mathrm{NC}$ siRNA; siSNHG12, cells transfected with siRNA targeting SNHG12; SNHG12, cells transfected with SNHG12 overexpression vector; Blank, cells transfected with empty vector. SNHG12, small nucleolar RNA host gene 12; siRNA, small interfering RNA; NC, negative control; miR, microRNA.

associated with lymph node metastasis, advanced clinical stage and poor overall survival of CRC patients.
Next, the exact roles of SNHG12 to regulate CRC cell proliferation and invasion were investigated. It was 
A

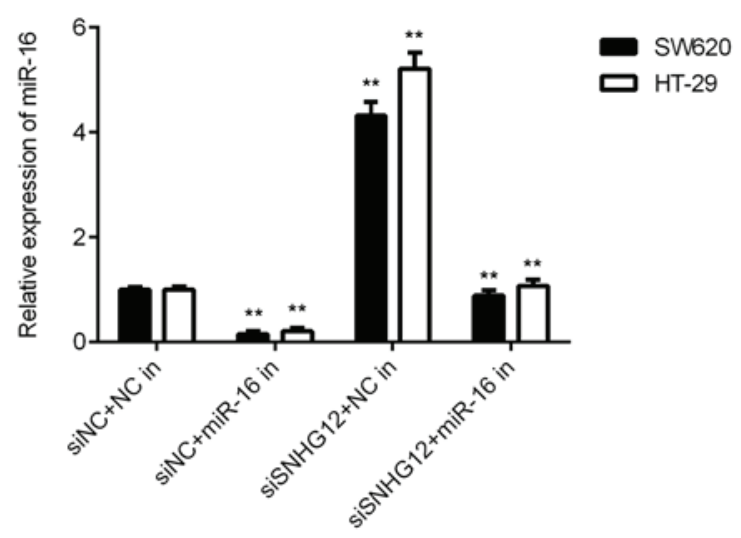

B
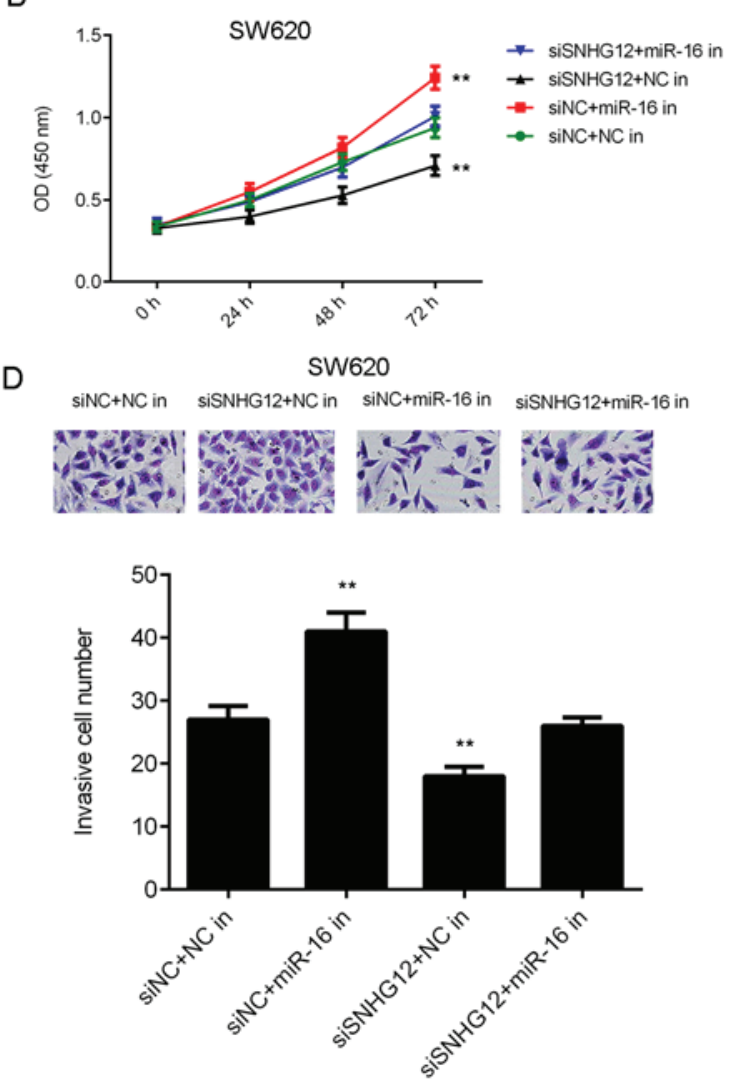

C
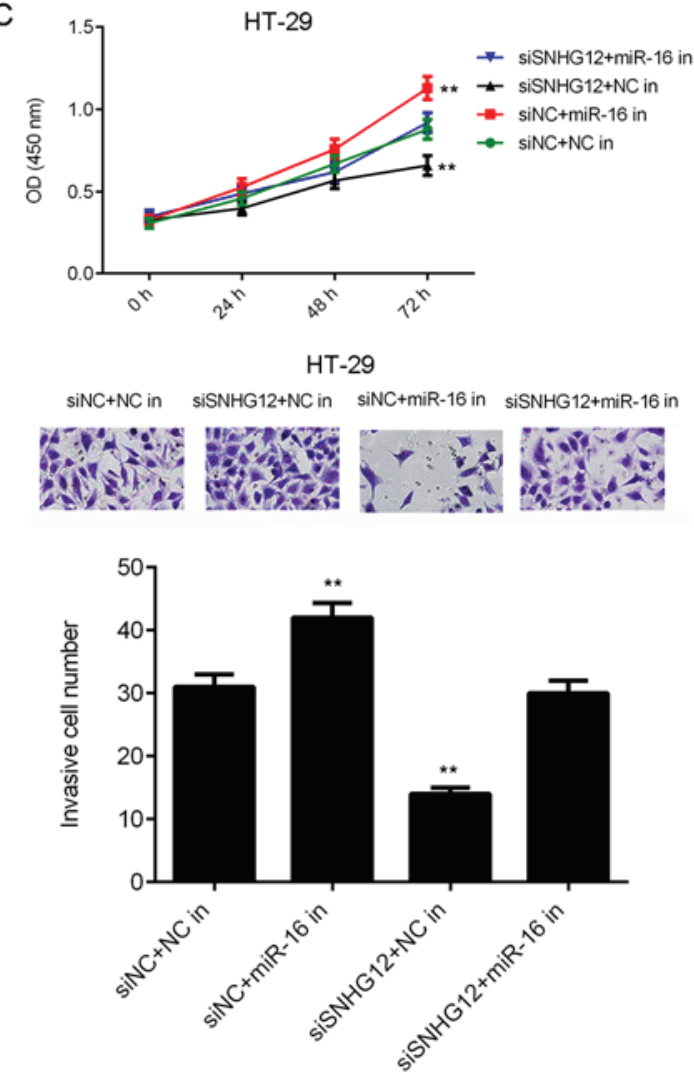

Figure 5. miR-16 is involved in SNHG12-mediated colorectal cancer cell proliferation and invasion. SW620 and HT-29 cells were co-transfected with NC siRNA and NC inhibitor (siNC+NC in), NC siRNA and miR-16 inhibitor (siNC+miR-16 in), SNHG12 siRNA and NC inhibitor (siSNHG12+NC in), or with SNHG12 siRNA and miR-16 inhibitor (siSNHG12+miR-16 in). After transfection, (A) reverse transcription-quantitative polymerase chain reaction was used to examine the levels of miR-16. CCK-8 assay using (B) SW620 and (C) HT-29 cells, and (D and E) a Transwell assay (magnification, x200) were performed to examine cell proliferation and invasion, respectively. ${ }^{* *} \mathrm{P}<0.01$ vs. siSNHG12+NC. siSNHG12, siRNA targeting SNHG12; in, inhibitor; SNHG12, small nucleolar RNA host gene 12; siRNA, small interfering RNA; NC, negative control; OD, optical density; miR, microRNA.

demonstrated that inhibition of SNHG12 expression led to a significant reduction in CRC cell proliferation and invasion. Consistent with these results, Wang et al (17) also reported that SNHG12 promoted CRC cell proliferation and that overexpression of SNHG12 enhanced the cell cycle progression of SW480 CRC cells, while knockdown of SNHG12 repressed the cell cycle progression of HT29 CRC cells. Furthermore, they demonstrated that SNHG12 also inhibited CRC cell apoptosis by increasing the expression of cell cycle-associated proteins and suppressing the expression of caspase 3 (17). These previous and the present results indicated that SNHG12 may have a role in promoting CRC proliferation and metastasis.

Next, the underlying mechanisms of the regulatory effect of SNHG12 on CRC cell proliferation and invasion were assessed in vitro. TargetScan software predicted that miR-16 and SNHG12 had complementary binding sites. To confirm this predicted binding interaction, the expression of miR-16 was examined in CRC, revealing that miR-16 was markedly downregulated in $\mathrm{CRC}$ tissues and cell lines. Furthermore, an inverse correlation was observed between miR-16 and SNHG12 in CRC tissues. To further clarify the association between miR-16 and SNHG12 in 
CRC, a luciferase reporter gene assay was performed, and the results indicated that transfection with miR-16 mimics markedly reduced the luciferase activity of WT SNHG12 reporter vector but did not affect the luciferase activity of MT SNHG12 in SW620 and HT-29 cells. These results suggest that SNHG12 may sponge miR-16 in CRC cells. As it was further observed that miR-16 expression was negatively regulated by SNHG12 in CRC cells, it was speculated that miR-16 is involved in SNHG12-mediated CRC cell proliferation and invasion, which was further confirmed by the result that inhibition of miR-16 expression abrogated the suppressive effects of SNHG12 downregulation on $\mathrm{CRC}$ cell proliferation and invasion.

In summary, the present study demonstrated that SNHG12 is upregulated in CRC and has a role in promoting CRC cell proliferation and invasion by acting as a sponge of miR-16. Thus, SNHG12 may be a promising therapeutic target for CRC.

\section{Acknowledgements}

Not applicable.

\section{Funding}

No funding was received.

\section{Availability of data and materials}

All data generated or analysed during the present study are included in this published article.

\section{Authors' contributions}

FR designed the study and wrote the manuscript. YL and J-YZ collected clinical tissues. SW, YS, J-PZ and FR performed the in-vitro experiments and statistical analysis.

\section{Ethics approval and consent to participate}

The present study was approved by the Research Ethics Committee of the Second Xiangya Hospital, Central South University (Changsha, China) and written informed consent had been obtained from all subjects.

\section{Patient consent for publication}

Not applicable.

\section{Competing interests}

The authors declare that they have no competing interests.

\section{References}

1. Siegel RL, Miller KD and Jemal A: Cancer statistics, 2015. CA Cancer J Clin 65: 5-29, 2015.

2. Torre LA, Bray F, Siegel RL, Ferlay J, Lortet-Tieulent J and Jemal A: Global cancer statistics, 2012. CA Cancer J Clin 65: 87-108, 2015

3. Li JW, Huang CZ, Li JH, Yuan JH, Chen QH, Zhang WF, Xu ZS, Liu YP, Li Y, Zhan MX and Lu LG: Knockdown of metadherin inhibits cell proliferation and migration in colorectal cancer. Oncol Rep 40: 2215-2223, 2018.
4. Cen C, Li J, Liu J, Yang M, Zhang T, Zuo Y, Lin C and Li X: Long noncoding RNA LINC01510 promotes the growth of colorectal cancer cells by modulating MET expression. Cancer Cell Int 18: 45, 2018.

5. Price TJ, Tang M, Gibbs P, Haller DG, Peeters M, Arnold D, Segelov E, Roy A, Tebbutt N, Pavlakis N, et al: Targeted therapy for metastatic colorectal cancer. Expert Rev Anticancer Ther 18: 991-1006, 2018.

6. Islam F, Gopalan V, Vider J, Wahab R, Ebrahimi F, Lu CT, Kasem K and Lam AKY: MicroRNA-186-5p overexpression modulates colon cancer growth by repressing the expression of the FAM134B tumour inhibitor. Exp Cell Res 357: 260-270, 2017.

7. Xiao Z, Qu Z, Chen Z, Fang Z, Zhou K, Huang Z, Guo X and Zhang Y: LncRNA HOTAIR is a prognostic biomarker for the proliferation and chemoresistance of colorectal cancer via MiR-203a-3p-mediated Wnt/ $\beta$-catenin signaling pathway. Cell Physiol Biochem 46: 1275-1285, 2018.

8. Xie B, Deng Z, Pan Y, Fu C, Fan S, Tao Y, Zhou J and Xiao D: Post-transcriptional regulation DPC4 gene by miR-190 in colorectal cancer cells. J Cancer Res Ther 14: 838-843, 2018.

9. Liu K, Yao H, Wen Y, Zhao H, Zhou N, Lei $S$ and Xiong L: Functional role of a long non-coding RNA LIFR-AS1/miR-29a/TNFAIP3 axis in colorectal cancer resistance to pohotodynamic therapy. Biochim Biophys Acta 1864: 2871-2880, 2018.

10. Liao D, Li T, Ye C, Zeng L, Li H, Pu X, Ding C, He Z and Huang GL: miR-221 inhibits autophagy and targets TP53INP1 in colorectal cancer cells. Exp Ther Med 15: 1712-1717, 2018.

11. Yunusov D, Anderson L, DaSilva LF, Wysocka J, Ezashi T, Roberts RM and Verjovski-Almeida S: HIPSTR and thousands of lncRNAs are heterogeneously expressed in human embryos, primordial germ cells and stable cell lines. Sci Rep 6: 32753, 2016.

12. Mercer TR, Dinger ME and Mattick JS: Long non-coding RNAs: Insights into functions. Nat Rev Genet 10: 155-159, 2009.

13. Zhou Y, Chen Y, Ding W, Hua Z, Wang L, Zhu Y, Qian H and Dai T: LncRNA UCA1 impacts cell proliferation, invasion, and migration of pancreatic cancer through regulating miR-96/FOXO3. IUBMB Life 70: 276-290, 2018.

14. Peng Z, Liu $\mathrm{C}$ and $\mathrm{Wu} \mathrm{M}$ : New insights into long noncoding RNAs and their roles in glioma. Mol Cancer 17: 61, 2018.

15. Smolle MA and Pichler M: The role of long non-coding RNAs in osteosarcoma. Noncoding RNA 4: E7, 2018.

16. Xu S, Kong D, Chen Q, Ping Y and Pang D: Oncogenic long noncoding RNA landscape in breast cancer. Mol Cancer 16: 129, 2017.

17. Wang JZ, Xu CL, Wu H and Shen SJ: LncRNA SNHG12 promotes cell growth and inhibits cell apoptosis in colorectal cancer cells. Braz J Med Biol Res 50: e6079, 2017.

18. Lan T, Ma W, Hong Z, Wu L, Chen X and Yuan Y: Long non-coding RNA small nucleolar RNA host gene 12 (SNHG12) promotes tumorigenesis and metastasis by targeting miR-199a/ b-5p in hepatocellular carcinoma. J Exp Clin Cancer Res 36: 11, 2017.

19. Ruan W, Wang P, Feng S, Xue Y and Li Y: Long non-coding RNA small nucleolar RNA host gene 12 (SNHG12) promotes cell proliferation and migration by upregulating angiomotin gene expression in human osteosarcoma cells. Tumour Biol 37: 4065-4073, 2016.

20. Lei W, Wang ZL, Feng HJ, Lin XD, Li CZ and Fan D: Long non-coding RNA SNHG12promotes the proliferation and migration of glioma cells by binding to HuR. Int J Oncol 53: 1374-1384, 2018.

21. Ding S, Qu W, Jiao Y, Zhang J, Zhang C and Dang S: LncRNA SNHG12 promotes the proliferation and metastasis of papillary thyroid carcinoma cells through regulating wnt/beta-catenin signaling pathway. Cancer Biomark 22: 217-226, 2018.

22. Yang $\mathrm{BF}, \mathrm{Cai} \mathrm{W}$ and Chen $\mathrm{B}$ : LncRNA SNHG12 regulated the proliferation of gastric carcinoma cell BGC-823 by targeting microRNA-199a/b-5p. Eur Rev Med Pharmacol Sci 22: 1297-1306, 2018.

23. Dong J, Wang Q, Li L and Xiao-Jin Z: Upregulation of long non-coding RNA small nucleolar RNA host gene 12 contributes to cell growth and invasion in cervical cancer by acting as a sponge for MiR-424-5p. Cell Physiol Biochem 45: 2086-2094, 2018.

24. Zhang $\mathrm{H}$ and Lu W: LncRNA SNHG12 regulates gastric cancer progression by acting as a molecular sponge of miR320. Mol Med Rep 17: 2743-2749, 2018. 
25. Wang O, Yang F, Liu Y, Lv L, Ma R, Chen C, Wang J, Tan Q, Cheng Y, Xia E, et al: C-MYC-induced upregulation of lncRNA SNHG12 regulates cell proliferation, apoptosis and migration in triple-negative breast cancer. Am J Transl Res 9: 533-545, 2017.

26. Ambros V: The functions of animal microRNAs. Nature 431: 350-355, 2004

27. Li Y, Sun Z, Liu B, Shan Y, Zhao L and Jia L: Tumor-suppressive miR-26a and miR-26b inhibit cell aggressiveness by regulating FUT4 in colorectal cancer. Cell Death Dis 8: e2892, 2017.

28. Lv H, Zhang Z, Wang Y, Li C, Gong W and Wang $X$ MicroRNA-92a promotes colorectal cancer cell growth and migration by inhibiting KLF4. Oncol Res 23: 283-290, 2016.

29. Qian J, Jiang B, Li M, Chen J and Fang M: Prognostic significance of microRNA-16 expression in human colorectal cancer. World J Surg 37: 2944-2949, 2013

30. Ma Q, Wang X, Li Z, Li B, Ma F, Peng L, Zhang Y, Xu A and Jiang B: microRNA-16 represses colorectal cancer cell growth in vitro by regulating the p53/survivin signaling pathway. Oncol Rep 29: 1652-1658, 2013.
31. Livak KJ and Schmittgen TD: Analysis of relative gene expression data using real-time quantitative PCR and the 2(-Delta Delta C(T)) method. Methods 25: 402-408, 2001

32. Wang P, Chen D, Ma H and Li Y: LncRNA SNHG12 contributes to multidrug resistance through activating the MAPK/Slug pathway by sponging miR-181a in non-small cell lung cancer. Oncotarget 8: 84086-84101, 2017.

33. Wang X, Qi G, Zhang J, Wu J, Zhou N, Li L and Ma J: Knockdown of long noncoding RNA small nucleolar RNA host gene 12 inhibits cell growth and induces apoptosis by upregulating miR-138 in nonsmall cell lung cancer. DNA Cell Biol 36: 892-900, 2017.

(c) (i) () This work is licensed under a Creative Commons Attribution-NonCommercial-NoDerivatives 4.0 International (CC BY-NC-ND 4.0) License. 